\title{
Constituent Quarks and the Spin of the Proton
}

\author{
H. Fritzsch and G. Eldahoumi \\ Physics Department \\ University of Munich, Germany
}

December 2, 2018

\begin{abstract}
The constituent quarks are interpreted as bound states, which have an internal structure. The quark distributions of the proton are related to those of the constituent quarks. The experiments support this hypothesis. Likewise the spin structure of the proton is related to the spin structure of the constituent quarks. We find that about $30 \%$ of the spin of a constituent quark is given by the valence quark, and $70 \%$ are provided by the gluons.
\end{abstract}

In the Standard Model the strong interactions are described by the gauge theory of quantum chromodynamics, introduced in 1972 (ref.1,2). In this theory quarks and gluons interact and form the baryons and mesons as bound states. Both the massive quarks and the massless gluons are permanently confined and do not appear as free particles.

The quarks were introduced in 1964 by M. Gell-Mann (ref.3) and G. Zweig (ref.4) as the constituents of the hadrons. In the deep inelastic scattering experiments of electrons and nucleons the quarks were observed indirectly at SLAC in 1968. But it was found that the nucleons consist not only of the three valence quarks, but also of many quark-antiquark pairs.

Today we distinguish between constituent quarks and current quarks, but it remained obscure what role the constituent quarks play in the theory of QCD. In particular the magnetic moments of the baryons in the baryon octet are described very well by the three constituent quarks, if one uses the $\mathrm{SU}(6)$-wave functions. For example, the ratio of the magnetic moments of the proton and neutron is predicted to be $-3 / 2$ (ref.5). The experiments give for this ratio 1.46, i.e. experiment and theory agree within $3 \%$. The magnetic moments of the other baryons in the $\mathrm{SU}(3)$-octet agree also very well with the model, if one adjusts the ratio of the quark masses $m_{s} / m_{u}$. Thus the constituent quarks seem to exist inside the baryons and mesons. Various long range properties of the hadrons, not only the magnetic moments, can be described rather well by the model of the constituent quarks.

Using the $\mathrm{SU}(6)$ wave functions for the nucleons, one can also calculate the ratio of the axial vector coupling constant, describing the beta decay, and the vector coupling constant :

$$
\frac{g_{A}}{g_{V}}=\frac{5}{3}
$$


However the experiments give for his ratio:

$$
\frac{g_{A}}{g_{V}}=1.2695 \pm 0.0029
$$

The experimental value is about $24 \%$ less than expected. The theoretical prediction follows, if the three quarks are in an s wave. If the space wave function has contributions from non-zero angular momenta (p-wave etc.), the ratio would be reduced. We shall assume that non-zero angular momenta are present and lead to the reduced value of the axial vector coupling. In this case the predictions for the ratios of the magnetic moments would still be valid.

We suggest that a hadron can be viewed as a loosely bound system of constituent quarks, which have an effective mass of about $330 \mathrm{MeV}$. However the constituent quarks are not pointlike objects, but composite systems, consisting of a valence quark, surrounded by a cloud of gluons and quark-antiquark pairs. The effective mass of a constituent quark is dynamically generated and is due to the cloud of gluons and pairs, surrounding the valence quark. In a good approximation the nucleon mass is the sum of the masses of the three constituent quarks.

The internal structure of the nucleons, measured in the deep inelastic scattering, can then be traced back to the internal structure of the constituent quarks, which we denote by the capital letters $U, D$ and $S$. The internal structure of the $U$-quark is given by quark and gluon distribution functions:

$$
u(x), \bar{u}(x), d(x), \bar{d}(x), s(x), \bar{s}(x), G(x)
$$

Thus a constituent $U$-quark depends on 7 functions (the heavy quarks c, b, t are neglected). The constituent $D$-quark is obtained after the interchange of $u$ and $d$.

The proton consists of two $U$-quarks and one $D$-quark: $P=(U U D)$. The current quark distribution functions of the proton are given by:

$$
\begin{aligned}
u_{p}(x) & =2 u(x)+d(x) \\
d_{p}(x) & =2 d(x)+u(x) \\
\bar{u}_{p}(x) & =2 \bar{u}(x)+\bar{d}(x) \\
\bar{d}_{p}(x) & =2 \bar{d}(x)+\bar{u}(x) \\
s_{p}(x) & =3 s(x) \\
\bar{s}_{p}(x) & =3 \bar{s}(x) \\
g_{p}(x) & =3 G(x)
\end{aligned}
$$

The current quark distributions of the $U$-quark have to obey the following sum rules:

$$
\begin{aligned}
& \int_{0}^{1} d x(u-\bar{u})=1 \\
& \int_{0}^{1} d x(d-\bar{d})=0 \\
& \int_{0}^{1} d x(s-\bar{s})=0
\end{aligned}
$$


Using the relations (4), we find for the current quark distributions of the $U$ quark:

$$
\begin{aligned}
u(x) & =\frac{1}{3}\left(2 u_{p}(x)-d_{p}(x)\right) \\
\bar{u}(x) & =\frac{1}{3}\left(2 \bar{u}_{p}(x)-\bar{d}_{p}(x)\right) \\
d(x) & =\frac{1}{3}\left(2 d_{p}(x)-u_{p}(x)\right) \\
\bar{d}(x) & =\frac{1}{3}\left(2 \bar{d}_{p}(x)-\bar{u}_{p}(x)\right) \\
s(x) & =\frac{1}{3} s_{p}(x) \\
\bar{s}(x) & =\frac{1}{3} \bar{s}_{p}(x) \\
G(x) & =\frac{1}{3} g_{p}(x)
\end{aligned}
$$

The sum of the contributions of the quarks and antiquarks to the nucleon momentum is about $45 \%$ (ref.6):

$$
\int_{0}^{1} x\left[\left(u_{p}+\bar{u}_{p}\right)+\left(d_{p}+\bar{d}_{p}\right)+\left(s_{p}+\bar{s}_{p}\right)\right] d x \cong 0.45
$$

It follows that the contributions of the gluons to the nucleon momentum must be about $55 \%$ :

$$
\int_{0}^{1} x g_{p}(x) d x \cong 0.55
$$

A constituent quark contributes $33 \%$ to the momentum of a nucleon. Thus for the distribution functions of the constituent quarks $u, d$, etc. we find:

$$
\begin{aligned}
& \int_{0}^{1} x[u+\bar{u}+d+\bar{d}+s+\bar{s}] d x \cong 0.15 \\
& \int_{0}^{1} x G(x) d x \cong 0.18
\end{aligned}
$$

If we would know the six quark and antiquark distribution functions of the proton in detail, we would be able to determine the six distribution functions of a constituent quark. However the distribution functions of the proton are not known in detail, in particular the small strange quark distribution function. We shall assume that we can neglect the strange quarks in the nucleon and set the strange distribution function equal to zero. We assume, as expected in QCD, that the $u$ and $d$ antiquarks are the same: $\bar{u}_{p}=\bar{d}_{p}$. Then we can express the distribution functions of the proton as follows:

$$
\begin{aligned}
& u_{p}=2 u+d \\
& d_{p}=2 d+u \\
& \bar{u}_{p}=\bar{d}_{p}
\end{aligned}
$$


Thus the quark distribution functions of the $U$-quark are given by:

$$
\begin{aligned}
u & =\frac{1}{3}\left(2 u_{p}-d_{p}\right) \\
d & =\frac{1}{3}\left(2 d_{p}-u_{p}\right) \\
\bar{u} & =\bar{d}
\end{aligned}
$$

The distribution function $d$ of a $U$-quark is expected to be equal to the antiquark distribution $\bar{d}=\bar{u}$. They can be neglected for $x>0.2$. Thus we expect that the function $d$ vanishes for $x>0.2$. Within the experimental errors the function $d$ vanishes.

We conclude that the constituent quarks appear as bound states of current quarks, antiquarks and gluons. They act as the constituents of the hadrons, but have their own substructure. The substructure of the nucleons, observed in the deep inelastic scattering, is in reality the substructure of the constituent quarks.

Subsequently we consider the spin structure of the constituent quarks and relate it to the spin structure of the nucleon. The spin vector $S_{\mu}$ of a proton is given by the matrix element of the axial-vector current:

$$
2 M S_{\mu}=\left\langle p, s\left|\bar{\psi} \gamma_{\mu} \gamma_{5} \psi\right| p, s\right\rangle
$$

Information about the quark "spin content" of the proton is obtained by the quark axial charges $\Delta q$ :

$$
\begin{gathered}
2 M S_{\mu} \Delta q=\left\langle p, s\left|\bar{q} \gamma_{\mu} \gamma_{5} q\right| p, s\right\rangle \\
(q: \text { quarkfield })
\end{gathered}
$$

The axial charges $\Delta u, \Delta d$ and $\Delta s$ can be written as linear combinations of the isovector, $\mathrm{SU}(3)$ octet and $\mathrm{SU}(3)$ singlet charges:

$$
\begin{aligned}
& g_{A}^{(3)}=\Delta u-\Delta d \\
& g_{A}^{(8)}=\Delta u+\Delta d-2 \Delta s \\
& g_{A}^{(0)}=\Delta u+\Delta d+\Delta s
\end{aligned}
$$

In the quark model $\Delta q$ is interpreted as the amount of spin, carried by the quarks and antiquarks of the flavor $q$.

The sum

$$
g_{A}^{(0)}=\triangle \Sigma=\triangle u+\triangle d+\triangle s
$$

has been measured (ref.7). It is much smaller than expected in the quark model.

$$
\triangle \Sigma \cong 0.30 \pm 0.1
$$

The nucleon spin can be decomposed into a quark contribution $\triangle \Sigma$, a gluon contribution $\triangle G$ and an orbital contribution $\triangle L$ :

$$
\frac{1}{2}=\frac{1}{2} \triangle \Sigma+\triangle G+\triangle L
$$


If we take $\triangle L=0, \triangle G$ would have to be about 0.35 .

We introduce the spin-dependent distribution functions $u_{+}, u_{-}$, etc. of the constituent quarks. The index "+" or "-" denotes the helicity of the corresponding quark or antiquark in a polarized $U$-quark with positive helicity. Especially we consider the integrals:

$$
\begin{aligned}
& \int_{0}^{1} d x\left[\left(q_{+}+\bar{q}_{+}\right)-\left(q_{-}+\bar{q}_{-}\right)\right]=I_{q} \\
& (q=u, d, s)
\end{aligned}
$$

The difference $\left(I_{u}-I_{d}\right)$ is the analogue of the Bjorken sum rule for the constituent quarks:

$$
I_{u}-I_{d}=g_{a}
$$

$g_{a}$ : axial vector coupling constant, given by the isotriplet axialvector current.

A polarized constituent $U$-quark depends in particular on the four functions $u_{+}, u_{-}, G_{+}$and $G_{-}$. If we would identify the constituent and current quarks and use a $\mathrm{SU}(6)$ wave function, we would have the unrealistic result:

$$
\begin{aligned}
& u_{+}=\delta(1-x) \\
& u_{-}=G_{+}=G_{-}=0
\end{aligned}
$$

The $U$-matrix element of the axialvector current $\left\langle U\left|\bar{u} \gamma_{\mu} \gamma_{5} u\right| U\right\rangle$ determines the integral $I_{u}$. In quantum chromodynamics the axial vector current is not conserved, if the quark masses are zero, due to the gluon anomaly (ref.2):

$$
\begin{aligned}
\partial^{\mu}\left(\bar{q} \gamma_{\mu} \gamma_{5} q\right) & =\frac{g^{2}}{16 \pi^{2}} \tilde{G} G \\
\tilde{G} G & =\sum_{a} \tilde{G}_{a}^{\mu \nu} G_{\mu \nu}^{a} \\
\tilde{G}_{a}^{\mu \nu} & =\frac{1}{2} \varepsilon_{\mu \nu \alpha \beta} G_{a}^{\alpha \beta}
\end{aligned}
$$

The isotriplet matrix element $\left\langle U\left|\left(\bar{u} \gamma_{\mu} \gamma_{5} u-\bar{d} \gamma_{\mu} \gamma_{5} d\right)\right| U\right\rangle$ determines the integral $\left(I_{u}-I_{d}\right)$. The corresponding isosinglet matrix element is given by the integral:

$$
\int_{0}^{1} d x\left[\left(u_{+}+\bar{u}_{+}-u_{-}-\bar{u}_{-}\right)+\left(d_{+}+\bar{d}_{+}-d_{-}-\bar{d}_{-}\right)\right]=\sigma
$$

The number $\sigma$ can be interpreted as the contribution of the $u$ and $d$ quarks to the $U$-spin.

For the sum $\sigma$ we expect the same suppression as for $\Delta \Sigma$ :

$$
\sigma \cong 0.30 \pm 0.01
$$

Note that in the SU(3) limit we would have

$$
d_{+}=s_{+}, d_{-}=s_{-}, \bar{d}_{+}=\bar{s}_{+}, \bar{d}_{-}=\bar{s}_{-}
$$


In a naive model, in which there is no difference between constituent and current quarks, a $U$-quark with positive helicity would consist only of a $u$-quark with positive helicity. Thus we would have:

$$
\int_{0}^{1} d x u_{+}=1
$$

$d_{+}=d_{-}=\bar{d}_{+}=\bar{d}_{-}=u_{-}=\bar{u}_{+}=\bar{u}_{-}=0$,

and we obtain:

$$
g_{a}=\sigma=+1
$$

Using a $\mathrm{SU}(6)$ wave function for the nucleon, we then find

$$
\frac{G_{A}}{G_{V}}=5 / 3
$$

( $G_{A}$ : axialvector coupling constant, $G_{V}$ :vector coupling constant)

In the experiments one finds

$$
\frac{G_{A}}{G_{V}}=1.2695 \pm 0.0029
$$

The $\mathrm{SU}(6)$ prediction is based on the assumption that the three quarks do not have orbital angular momentum(s-wave). We attribute the difference between the $\mathrm{SU}(6)$-prediction (25) and the experimental result (26) to contributions from angular momenta ( $p$-wave etc.).

The reduction (ratio of the observed value of $\frac{G_{A}}{G_{V}}$ and the theoretical value) is about $24 \%$. The same reduction is expected for $\triangle \Sigma$, i.e. we would expect $\triangle \Sigma \approx 0.76$. In reality $\Delta \Sigma$ is about 0.30 , i.e. there is a further reduction of $60 \%$ from 0.76 to 0.30 . In our constituent quark model the some reduction should present in the constituent quarks: $\sigma \approx 0.40$.

This reduction should be the result of the gluon anomaly. The isotriplet matrix element $g_{a}$ is not affected by the gluon anomaly.

The integral

$$
\int_{0}^{1} d x\left[\left(d_{+}+\bar{d}_{+}-d_{-}-\bar{d}_{-}\right) \cong \frac{1}{3}\left(\sigma-g_{a}\right)\right.
$$

is non-zero due to the gluon anomaly. It is given by the matrix element $\left\langle U\left|\bar{d} \gamma_{\mu} \gamma_{5} d\right| U\right\rangle$. In the naive quark model this matrix element vanishes. We find, taking $\sigma \cong 0.40 \pm 0.13$ :

$$
\begin{aligned}
& \int_{0}^{1} d x\left[\left(d_{+}+\bar{d}_{+}-d_{-}-\bar{d}_{-}\right) \cong \frac{1}{3}\left(\sigma-g_{a}\right) \cong-0.2 \pm 0.04\right. \\
& \int_{0}^{1} d x\left[\left(u_{+}+\bar{u}_{+}-u_{-}-\bar{u}_{-}\right)=\frac{1}{3}\left(\sigma+2 g_{a}\right) \cong 0.80 \pm 0.04\right.
\end{aligned}
$$


Due to the QCD anomaly $\bar{q} q$-pairs are created inside the $U$-state. The pairs are polarized and reduce the contribution of the quarks to the $U$-spin. According to the $d$-integral given above the sum $\left(d_{-}+\bar{d}_{-}\right)$must be different from zero, but the sum $\left(d_{+}+\bar{d}_{+}\right)$might vanish. The QCD anomaly does not distinguish between $u$ and $d$ quarks. Thus $\bar{u} u$ pairs and $\bar{d} d$ pairs are created with equal strength, and we expect $d_{-}=\bar{d}_{-}=\bar{u}_{-}=u_{-}$.

A constituent $U$-quark can be viewed as a valence $u$-quark, surrounded by $\bar{q} q$-pairs, which reduce the spin contribution of the quarks significantly. It has $\operatorname{spin} \frac{1}{2}$ :

$$
\frac{1}{2}=\frac{1}{2} \cdot \sigma+\Delta G
$$

( $\Delta G$ : gluon contribution to the spin of the constituent quark)

Using the quoted value of $\sigma$, we can calculate $\Delta G$ :

$$
\Delta G=\frac{1}{2}(1-\sigma) \cong 0.30 \pm 0.06
$$

In this calculation we have used the $\mathrm{SU}(3)$ symmetry. Any SU(3)-breaking would reduce the integral over the strange quark densities. If we neglect the strange quarks $\left(s_{+}=s_{-}=\bar{s}_{+}=\bar{s}_{-}=0\right)$, we would find:

$$
\Delta G \cong 0.35 \pm 0.06,
$$

thus the gluonic contribution would increase slightly.

Using eq.(30), we find that about $40 \%$ of the spin of a constituent quark is provided by the quarks, and $60 \%$ is provided by gluons. A polarized constituent quark consists of a polarized valence quark, surrounded by polarized quarkantiquark pairs and by a cloud of polarized gluons, which provide a large part of the spin.

In the simple $\mathrm{SU}(6)$ model about $24 \%$ of the nucleon spin is due to orbital angular momenta of the three quarks, and $76 \%$ is due to the spins of the quarks. The nucleon spin $1 / 2$ is given by:

$$
\frac{1}{2}=\left(\frac{1}{2} \times 0.76\right)+\left(\frac{1}{2} \times 0.24\right)
$$

In our model we find:

$$
\begin{aligned}
\frac{1}{2} & =\frac{1}{2} \Delta \Sigma+\Delta G+\Delta L \\
& =\left(\frac{1}{2} \times 0.30\right)+\left(\frac{1}{2} \times 0.46\right)+\left(\frac{1}{2} \times 0.24\right)
\end{aligned}
$$

About $24 \%$ of the nucleon spin is due to orbital angular momenta, about $46 \%$ is due to the spins of the gluons inside the constituent quarks, and only $30 \%$ is due to the spins of the quarks.

\section{References}

[1] H. Fritzsch and M. Gell-Mann, eConf C720906 V2 135-165, 1972. Also in Physics, Proceedings of the XVI International Conference on High Chicago 1972 p.135 (J. D. Jackson, A. Roberts, eds.).e-Print: hep-ph/0208010. 
[2] H. Fritzsch, M. Gell-Mann and H. Leutwyler, Phys.Lett. B47 365-368,1973.

[3] M. Gell-Mann, Phys.Lett. 8:214-215,1964.

[4] G. Zweig, An SU(3) Model for Strong Interaction Symmetry and ist Breaking, parts I and II, CERN preprints 8182 TH. 401 and TH. 402, 1964.

[5] M. A. B. Beg, B. W. Lee and A. Pais, Phys. Rev. Lett. 13 (1964) 514.

[6] See e.g. C.Quigg, Gauge Theories of Strong, Weak, and Electromagnetic Interactions, p. 174.

[7] For a review see: S. Bass, Rev. Mod. Phys. 77 (2005) 1257. 